were absent in both lower extremities. Babinski's signs were not noted. The Romberg sign was present. Magnetic resonans imaging (MRI) of the spinal cord revealed enhancing $T_{2}$ hyperintensity within the spinal cord, from $T_{10}-L_{1}$. (Figure 1). Cerebrospinal fluid (CSF) showed no pleocytosis and normal protein and glucose concentrations. The CSF did not show oligoclonal banding and immunglobulin (Ig)G index was normal. Serum serologic evaluation of Borrelia burgdorferi was positive for IgM but negative for IgG. CSF serologic evaluation was negative (both ELISA and Western blot). The patient was diagnosed as manifesting acute transverse myelitis. He was treated with intravenous methylprednisolone pulse therapy (1000 mg/day for 5 consecutive days), followed by oral prednisolone (1 mg/kg per day). After the start of steroid therapy, the patient showed gradual clinical improvement and was able to walk on the 30 th day of illness. We also administered doxycycline $4 \mathrm{mg} / \mathrm{kg}$ per day for 14-days for borreliosis.

Conclusion This case serves as a reminder that acute transverse myelitis can be a rare clinical manifestation of Lyme disease.

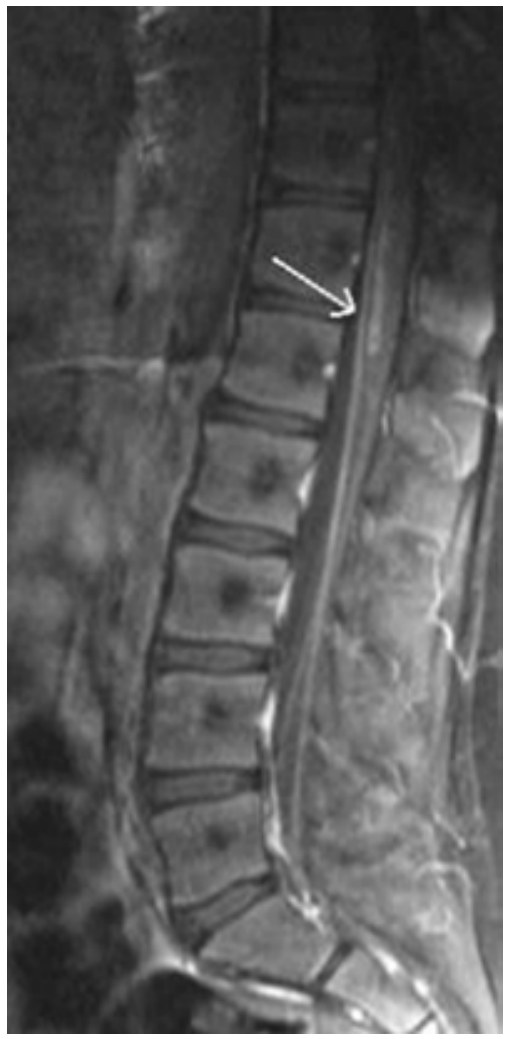

Abstract 832 Figure 1

\section{THE EFFECT OF PROBIOTICS ON COLONIZATION OF RESISTANT ORGANISMS IN PRETERM INFANTS}

doi:10.1136/archdischild-2012-302724.0833

${ }^{1} A$ Kurt, ${ }^{1} A$ Ecevit, $' D$ Anuk Ince, ${ }^{1} A$ Abbasoglu, ${ }^{2} 0$ Kurt Azap, 'Z Ecevit, ${ }^{3} E$ Ogus, 'A Tarcan. 'Pediatrics; 'Infectious Diseases; ${ }^{3}$ Biostatistics, Baskent University Faculty of Medicine, Ankara, Turkey

Background and Aims To investigate the effect of probiotics on colonization of resistant organisms in preterm infants receiving antibiotics.

Methods This study comprised of preterm infants who were born $<36$ weeks and received antibiotic treatment or prophylaxis. Preterm infants were divided into two groups according to receiving probiotic (Lactobacillus reuteri). Stool culture and nasal swab culture were taken to determine colonization.
Results In the group receiving probiotics, mostly colonizing the stool cultures bacteries were Klebsiella spp, Escherichia coli, Enterococcus spp., Enterobacteriae spp., Staphylococcus spp. respectively, and in the group not receiving probiotic mostly colonizing the stool cultures bacteries were Klebsiella spp, Enterococcus spp., Staphylococcus spp., Escherichia coli, Enterobacteriae spp. respectively. When probiotic receiving group compared was with not receiving group, proliferation rate of stool cultures was higher in probiotic group. In the groups receiving and not receiving probiotic, proliferation of the nose cultures were similar. Increase in the proliferation rates of weekly stool cultures in probiotic receiving group was statistically significant but there was no statistically difference in the proliferation rates of nose and other cultures that were taken weekly. There was no statistical difference in both groups in the development of resistant organisms.

Conclusions The use of probiotics in neonatal intensive care unit for premature infants who received treatment with antibiotics, did not prevent the colonization of pathogenic microorganisms.

\section{THE SENSITIVITY OF BACTERIAL MENINGITIS SCORE IN CHILDREN WITH ACUTE MENINGITIS IN OATAR}

doi:10.1136/archdischild-2012-302724.0834

${ }^{1} \mathrm{H}$ Abdlrahman, ${ }^{1} \mathrm{~A}$ Alhamadi, ${ }^{1} \mathrm{~K}$ Al-Saddi, ${ }^{1} \mathrm{~W}$ Seleem, ${ }^{1} \mathrm{H}$ Badran, ${ }^{1} \mathrm{~N}$ Sharaf, ${ }^{2} \mathrm{~A}$ Farooq. ${ }^{1}$ Hamad Medical Center; ${ }^{2}$ Aspitar Medical Center, Doha, Oatar

Background The empirical use of antibiotics in children with suspected meningitis is a common clinical practice worldwide that often leads to drug resistance. It is difficult to clinically differentiate bacterial when compared to viral meningitis until a culture study ofcerebral spinal fluid (CSF) Or CSF viral PCR study is performed. A 'wait and see' approach may lead to undesirable outcome. Bacterial Meningitis Score (BMS) is a tool that was developed to help physicians to differentiate between viral versus bacterial meningitis.

Aim To determine the usefulness if any of BMS for discriminating between bacterial or viral meningitis is young children.

Methodology We retrospectively reviewed the charts of all children (from birth till 14 years old) who were admitted with the diagnosis of meningitis to Hamad general hospital in last 2 years period. Result A total 120 patients (68\% boys) with confirmed meningitis were reviewed during the study period. The mean age was $(6.3 \pm 2.7$ year). The majority of patients $112(93.3 \%)$ had viral type meningitis while the remaining had bacterial meningitis (Strep Pneumia, Neisseria meningitis and $\mathrm{H}$. Influenza). The sensitivity of BMS tool revealed a sensitivity of $100 \%$ (95\% CI: 75.1 to 100.0$)$ and a specificity of $60.9 \%$ (95\% CI:50.1-69.7).

Conclusion Our study shows that BMS is a simple, easy and highly sensitive tool that can differentiate bacterial from viral meningitis and it is use may limit the use of unnecessary antibiotic s and hospitalizations.

\section{THE VALUE OF A POSITIVE NITRATE TEST IN ROUTINE URINE ANALYSIS FOR THE DIAGNOSIS OF CHILDHOOD UTI}

doi:10.1136/archdischild-2012-302724.0835

HA Bin-Nakhi, E Bu-Hamrah, S Sadeq, AR Moodambail. Pediatrics, Adan Hospital, Kuwait, Kuwait

Urinary tract infection (UTI) is one of the most common diseases in children. Its diagnosis depends mainly on urine culture. Urinalysis is used as a screening test to exclude UTI. A hospital based, prospective case-review study was carried out at the Pediatric Department, Al-Adan Hospital, State of Kuwait. The objectives of this study is:

1. To determine the proportion of positive urine cultures identified in patient less than 12 years of age, admitted to hospital and was found to have a positive nitrite test in the routine urinanalysis. 
2. Furthermore, to study the confound factors which increase the sensitivity of this test in order to reduce unnecessary culture of urinanalysis.

A total of eighty patients admitted to Pediatrics wards at AlAdan Hospital over a period of four months were found to have a positive nitrite test in routine urinanalysis. only $38(47 \%)$ were significantly positive (pure bacterial growth of $>=100000 \mathrm{CFU} / \mathrm{ml}$ ) Other studied confound factors such as fever, urinary symptoms, rigors, family history and past history of UTI, history of nocturnal enuresis or constipation were only significant if taken in combination (of those with positive urine cultures $97 \%$ had more than one factor). Urinary leukocyte count was significantly elevated ( $>10 /$ hpf) in $90 \%$ of the patients with a positive urine cultures. Our data indicates that ordering urine culture in patients with a positive nitrite in urinanalysis should be restricted only to those who also have elevated urinary leukocyte esterase and those who have one or more risk factor for UTI.

\section{DISTRIBUTION OF INTESTINAL PARASYTES IN A PEDIATRICS CLINIC IN 3 YEARS' PERIOD}

doi:10.1136/archdischild-2012-302724.0836

${ }^{1} \mathrm{M}$ Ugras, ${ }^{20}$ Miman, ${ }^{3} \mathrm{~F}$ Alpay. ${ }^{1}$ Pediatrics, Yeditepe Univ Medical Faculty, Istanbul; ${ }^{2}$ Microbiology, ${ }^{3}$ Pediatrics, Kocatepe University Medical Faculty, Afyonkarahisar, Turkey

Aim Intestinal parasitic infections are frequently seen in developing countries. Clinical findings such as abdominal pain, anal itching, salivation during sleep, and nazal itching are related with the prevalence of parasitic infection. The reported ranges from different cities in Turkey vary between $4.4 \%$ and $44.6 \%$. We aimed to look for the prevalence of intestinal parasytes in children who were brought to our clinic in a 3-years' period.

Material and method The laboratory and clinical data of the children who were admitted to the Department of Pediatrics between January 2010 and December 2012 were retrospectively evaluated. Age, major complaint of the children were noted.

Results A total of 1790 stool samples were studied and 116 samples $(6.48 \%)$ had intestinal parasites. There were 1712 children aged between 5 month and 17 years. The complaints were abdominal pain, failure to thrive and anal itching. Intestinal parasites were Giardia intestinalis 33 (1.84\%), Blastocystis hominis 52 (2.9\%), Enterobius vermicularis 13 (0.72\%), Entamoeba coli 17 (0.95\%), Maya was found in 95 stool samples (5.3\%). Multiple parasites were seen in 1 (0.05\%) sample (Blastocystis hominis, Entamoeba coli).

Conclusion Intestinal parsites are frequently seen in developing countries. The mean vales are reported as $12 \%, 10 \%$ and $19 \%$ from different cities in Turkey, ranging between $4.4 \%$ and $44.6 \%$. We found $6.5 \%$ intestinal parasites among children. The most common parasite was $B$. hominis and $G$. intestinalis. The prevalence and type of the parasites are similar when compared with the studies made in our country.

\section{VIEWS OF HEALTH CARE STAFF ABOUT PANDEMIC INFLUENZA VACCINE PRIOR TO PANDEMICS}

doi:10.1136/archdischild-2012-302724.0837

${ }^{1} \mathrm{~A}$ Ozkaya-Parlakay, ${ }^{1} \mathrm{AH}$ Abdulmumin, ${ }^{1} \mathrm{~A}$ Kara, ${ }^{1} \mathrm{AB}$ Cengiz, ${ }^{2} \mathrm{E}$ Ince, ${ }^{2} \mathrm{E}$ Ciftci, ${ }^{3} \mathrm{H}$ Tezer, ${ }^{2} \mathrm{H}$ Ozdemir, ${ }^{3} \mathrm{E}$ Celiker. ${ }^{1}$ Pediatric Infectious Diseases, Hacetepe University, Faculty of Medicine; ${ }^{2}$ Pediatric Infectious Diseases, Ankara University; ${ }^{3}$ Pediatric Infectious Diseases, Ankara Hematology and Oncology Training Hospital, Ankara, Turkey

Pandemic influenza vaccine, is important in many aspects for health care staff. Our questionnaire was conducted in 3 different centers to evaluate view of health care staff for pandemic influenza vaccine prior to epidemics. Our questionnaire was conducted with health care workers having possibility of direct exposure to patients including 182 doctors, 158 nurses and 54 management staff with a total of
394 people. The center of the staff, position, period of Office, whether she/he had seasonal influenza vaccine, whether he/she plans to get pandemic influenza vaccine and reason for this was questioned and noted. Of the 394 healtcare staff included in our study 221 were working in Hacettepe University Hospital,102 were in Ankara University Hospital, 71 were in Ankara Hematology and Oncology Hospital. Of the people enrolled in our study 259 were planning to get pandemic influenza vaccine while 135 were not. Of the staff planning to get pandemic influenza vaccine $77.6 \%$ were planning because they thought they were in risc group, $22.4 \%$ were planning because there was pandemics. Of the staff not planning to get pandemic influenza vaccine $23 \%$ were not taking vaccine because they did not want be guinae pig, 30.9\% were concerned with side affects of vaccine, $46 \%$ had concerns as vaccine was too new. In case of group vaccination declaration of the specialists and determination of health care authorities is very important, so declarations about vaccine should adhere to this aspect. Other wise even in risc groups acceptance of the vaccine would be in lower rates.

\section{ABDOMINAL TUBERCULOSIS DUE TO MYCOBACTERIUM BOVIS}

doi:10.1136/archdischild-2012-302724.0838

G Lozano Sánchez, E Núñez Cuadros, D Moreno Pérez, VM Candón Moreno, B Carazo Gallego, A Urda Cardona. Paediatric, Hospital Carlos Hava, Málaga, Spain

Background Currently, disease related to Mycobacterium bovis $(\mathrm{MB}$ ) is rare in Spain (less than 1\% of tuberculosis cases). However it's more frequent in undeveloped countries where the way to become infected is usually by raw milk consumption.

Methods We describe the epidemiologic, clinical and therapeutic characteristics of 5 patients with abdominal tuberculosis due to $\mathrm{MB}$.

Results Five children ( 3 male) emanating from Morocco with ages between 2 and 6 years, all of them had fever, abdominal pain and constitutional syndrome. Three of them used to drink raw goat's or cow's milk. In 2 cases tuberculin skin test was over than $12 \mathrm{~mm}$. There was open surgery (3) or laparoscopy (2) for realization of lymph node biopsy or drainage of abscess. The diagnosis was through culture and PCR in drained material and/or lymph node. Associated complications were: intraabdominal bacterial infection (4), esteroraceus fistula (2), intestinal occlusion (3). All patients were immunocompetent, except one case with histocompatibility complex class II molecules deficiency. Empirical treatment consisted in isoniazid and rifampicin for 6 months, amikacin (3) or ethambutol (2) during 1 or 2 months respectively. One patient developed a Dress syndrome related to rifampicin. All patients received antituberculous drugs during 6 months, except two patients who needed extended therapy.

Conclusion Treatment for abdominal tuberculosis must be medical, and surgery should be used only in serious complications or biopsy. Although the clinical presentation is often very similar from the produced by $M$. tuberculosis, empirical therapy avoiding pyrazinamide should be started if $M B$ is suspected.

\section{VIEW OF PREGNANT WOMEN ABOUT PANDEMIC INFLUENZA VACCINE PRIOR TO PANDEMIA}

doi:10.1136/archdischild-2012-302724.0839

'A Ozkaya-Parlakay, ${ }^{20}$ Kara, ${ }^{1} \mathrm{~A}$ Kara, ${ }^{20}$ Ozyuncu. 'Pediatric Infectious Diseases, 2Ubstetrics and Gynecoligy, Hacetepe University, Faculty of Medicine, Ankara, Turkey

Afterwards pregnant women were detected to have serious complications with pandemic A (H1N1) virus in 2009, priority of their vaccination was accepted. Thus our study was planned to estimate the view of pregnant women about pandemic influenza vaccine to prevent disease admitting to a reference hospital having patients all 